\title{
Fuzzy Logic Based Real Time Obstacle Avoidance Controller for a Simplified Model of Hexapod Walking Robot
}

\author{
Umar Farooq, Muhammad Usman Asad, Muhammad Amar, Athar Hanif, and Syed Omar Saleh
}

\begin{abstract}
This paper describes the design of an intelligent controller based on fuzzy logic for hurdle avoidance task in hexapod walking robot. The controller takes input from two ultrasonic sensors mounted in front of the robot. The outputs from the controller are translated into servo motor commands for movement of legs. The controller is designed using Fuzzy Logic Toolbox of MATLAB and its implementation is realized with a cheap, readily available AT89C52 microcontroller. Experimental results obtained after testing the controller in an environment containing obstacles has proved its validity. The design is low cost and can be used as demonstration tool for teaching concepts of soft computing algorithms.
\end{abstract}

Index Terms - Fuzzy logic controller, hexapod walking robot, SRF04 ultrasonic sensors, AT89C52 microcontroller.

\section{INTRODUCTION}

Legged robots are important alternative to driving (wheeled) robots, since the majority of the world's land area is unpaved. Although driving robots are more specialized and better adapted to flat surfaces, as they can drive faster and navigate with higher precision, walking robots can be employed in environments that are more general. Walking robots follow nature by being able to navigate rough terrain or even climb stairs or over obstacles in a standard household situation, which could rule out most driving robots [1]. In recent years, a number of robotics engineers have taken an interest in building legged robots whose designs are based at least in part on biological principles of structure or control [2] The fundamental reason for the interest in biology is that legged animals can easily outperform the most agile robot over rough or irregular terrain. The work has been done on several designs which mimic the biological system of an insect. Examples are ant-like, cockroach-like or even spider-like robots [3].

Legged motion can easily avoid large obstacles on the path and any kind of direction change can be performed more quickly in less space. They can also move side-ways and can

Manuscript received June 27, 2013; revised September 15, 2013.

Umar Farooq, Muhammad Amar, and Syed Omar Saleh are with Department of Electrical Engineering, University of The Punjab Lahore (e-mail: engr.umarfarooq@yahoo.com, amar.ete611@yahoo.com, omar_saleh81@yahoo.com).

Muhammad Usman Asad and Athar Hanif are with Department of Electrical Engineering, The University of Lahore (e-mail: usmanasad01@hotmail.com, athar.hanif@ee.uol.edu.pk). represent a better approach for moving in environments, where the surface has less adherence (on the moon for instance, where the lower gravity causes a friction reduction). On the other hand, it is a hard task to let a hexapod move, because of the complexity of the robots kinematics and dynamics and the complex coordination between legs for each gait. A gait is defined as a sequence of leg motions coordinated with a sequence of body motions for the purpose of transporting the body of the legged robot from one place to another [4]. Furthermore, legged robots have to be designed to handle with obstacles, hill, stairs, and leg coordination in such environments is a tough task [5], [6].

This paper describes the design of a fuzzy logic controller for obstacle avoidance in simplified model of hexapod walking robot, driven by three servo motors and using alternating tripod gait mechanism for its locomotion. Fuzzy logic controller is selected due to its inherent ability to handle the imperfect and uncertain data provided by the sensors avoiding further processing of data. Also, the fuzzy inference system is a universal approximator, and therefore has the ability to do any non-linear mapping between sensor data and the control variables. The designed controller accepts range inputs from two ultrasonic sensors and generates movement commands for servo motors. The controller is designed in MATLAB and its rule base is optimized for best possible results. The controller is then implemented using a low cost, readily available AT89C52 microcontroller. Due to its simplicity and low cost, the design can be quickly constructed and used as educational tool for demonstrating soft computing algorithms in real time.

\section{PLATFORM DESIGN}

\section{A. Mechanical Hardware}

Fig. 1 shows the schematic view of the mechanical assembly. The robot design uses three servos, HS311 to achieve locomotion. The servo positioned in the middle of the body is attached to legs 2 and 5 and is used to rock the body back and forth and in turn lifts up legs 1, 3, 5 or legs 2, 4, 6 . Legs 1, 3 and 4, 6 are each controlled by one servo and move together via a mechanical linkage [7]. The completed version of the hexapod is shown in Fig. 2.

\section{B. Electronic Hardware}

1) Ultrasonic Sensors: Two SRF04 ultrasonic sensors are mounted to acquire 'distance to hurdle' information on left and right sides of the hexapod. They are placed sufficiently high from the ground to ensure that the cone-shaped coverage 
zone does not give false reading. The maximum range of sensors is set at $3 \mathrm{ft}$ corresponding to register count of 100 , but it is adjustable. More number of sensors can be added but it will increase the computational burden on microcontroller resulting in more complex hardware design.

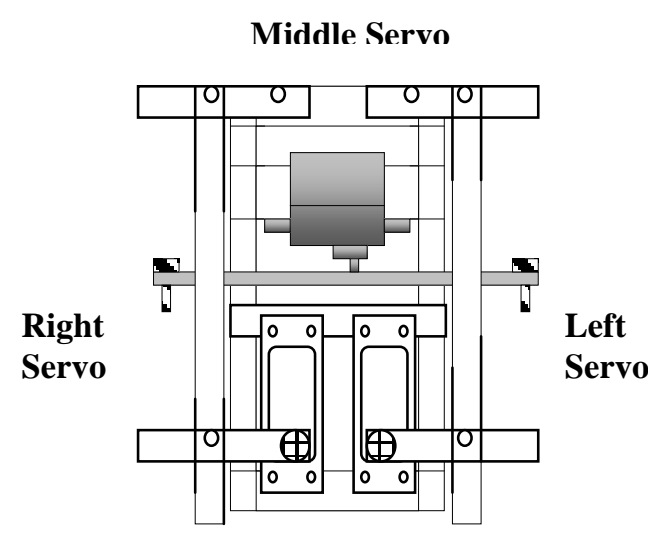

Fig. 1. Schematic View of Hexapod.

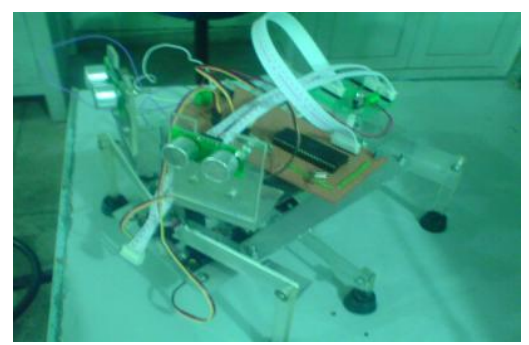

Fig. 2. Complete Version of Hexapod.

2) Microcontroller: Single chip 8-bit AT89C52 microcontroller having $8 \mathrm{~Kb}$ FLASH, 256 bytes RAM and 6 interrupt sources is selected as brain of hexapod. The microcontroller is responsible for reading sensors data (using timer0 interrupt service), executing fuzzy control algorithm and generating servo motor commands (using timer 1 interrupt service). The microcontroller is programmed in C-language using Keil $\mu$-Vision development platform.

\section{Walking Sequence}

The hexapod can walk forward, backward, turn left and right. However, the walking sequence for moving backward, turning left and turning right can be derived from forward walking sequence.

To start the forward walking sequence, all the legs are flat on ground with the servos in their middle position as shown in frame 1 of Fig. 3. The first move shown in frame 2 of Fig. 3 is to move leg 2 down, which lifts legs 1, 3, and 5 up off the ground. In frame3, leg 2 remains down and is used as a swivel as legs 4 and 6 are moved backwards, propelling the right side of the robot forward. In frame 4, leg 2 remains down while legs 1 and 3 are moved forward in anticipation of the next move. In frame 5, leg 5 is now moved down, lifting legs 2, 4, and 6 up off the ground. In frame 6 , leg 5 remains down and legs 4 and 6 are moved forward in anticipation of the next move. In frame 7 , leg 5 remains down and acts as a swivel as legs 1 and 3 are moved backwards, propelling the left side of the robot forward. For continuous forward walking, sequence is repeated from frames 3 to 7 [7].

For turning towards left, left servo motor is driven with small step than right servo motor and for turning towards right, right servo is driven with small step than left servo motor.

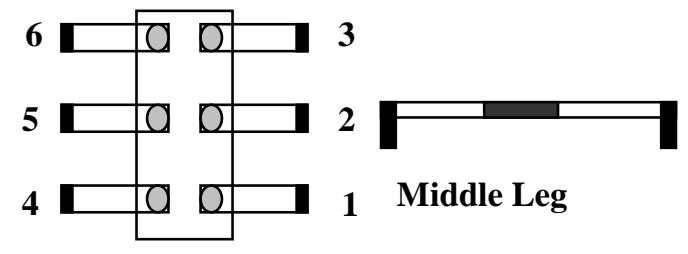

(a)
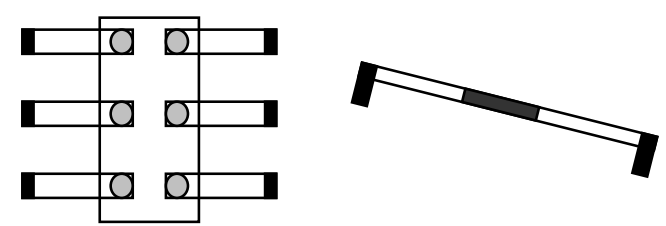

(b)
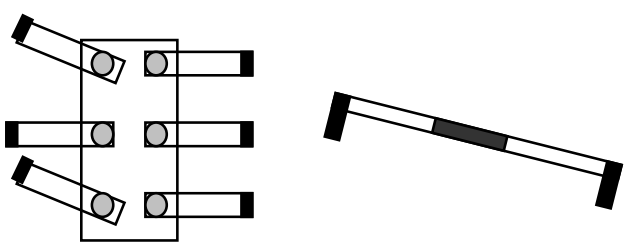

(c)
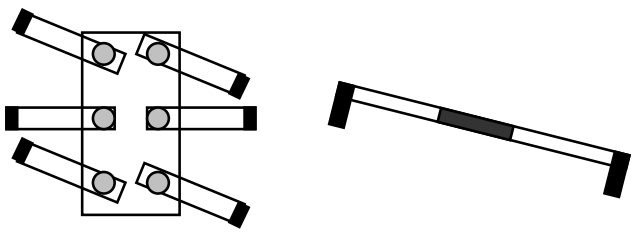

(d)
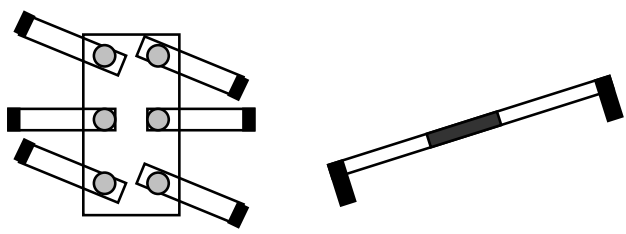

(e)
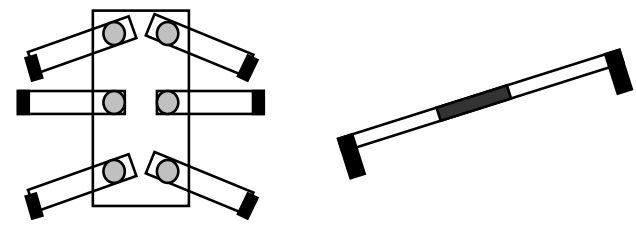

(f)
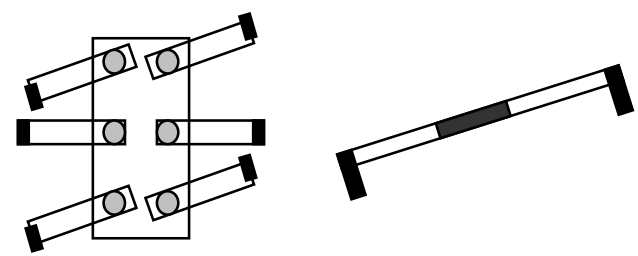

(g)

Fig. 3. (a) Initialization Step (b)-(g) Steps for Straight Walking Sequence

\section{FUZZY CONTROLLER DESIGN}

MATLAB® Fuzzy Logic Toolbox is used to aid in fuzzy logic controller (FLC) design. The toolbox contains functions, graphical user interfaces and data structures that allow the user to quickly design, test, simulate and modify a fuzzy 
inference system [8]. The steps in FLC design are described in this section.

\section{A. Fuzzification}

The inputs to FLC i.e., the range information from left sensor (L-SENSOR) and right sensor (R-SENSOR) is described by three fuzzy sets, Near, Med and Far. The membership functions for L-SENSOR (same for R-SENSOR), as shown in Fig. 4, are described by the expressions:

$$
\mu_{L-S E N S O R, N E A R}=\left\{\begin{array}{lc}
-2 l_{s}+100, & 0 \leq l_{s} \leq 50 \\
0, & l_{s} \geq 50
\end{array}\right.
$$

Fig. 4. Input Membership Function.

In these expressions, degree of belongingness of a particular linguistic variable is scaled in range $[0,100]$ to prevent floating point storage and calculations. Also, triangular membership function (MF) is used for all fuzzy sets because of limited computational resources of microcontroller. The outputs of FLC i.e., left servo motor (L-SERVO) and right servo motor (R-SERVO) positions are also described by three fuzzy sets: Small Step (S-STEP), Medium Step (M-STEP) and Large Step (L-STEP); the universe of discourse being from zero (minimum) to hundred (maximum) which describes the duty cycle values ranging from $10 \%$ to $20 \%$ of $50 \mathrm{~Hz}$ PWM signal for controlling the position of servos. The membership function description of L-SERVO and R-SERVO is similar to L-SENSOR or R-SENSOR as shown in Fig. 5.

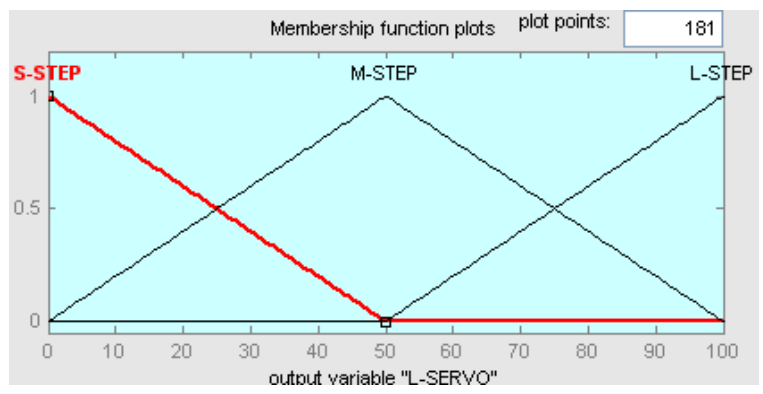

Fig. 5. Output Membership Function.

\section{B. Fuzzy Rule Base}

For avoiding the obstacles successfully, a total of nine rules are designed. These rules establish the relation between sensor values and motor positions in terms of linguistic values. A control surface is then plotted to visualize the variation in motor positions given the sensor values. The rule base for left and right servo motor position control is shown in Table I and Table II respectively while their control surfaces are shown in Fig. 6 and Fig. 7 respectively.

TABLE I: Rule BASE FOR CHANGE IN LEFT SERVO POSITION

\begin{tabular}{cccc}
\hline \hline L-SENSOR/ & NEAR & MED & FAR \\
R-SENSOR & L-STEP & S-STEP & S-STEP \\
NEAR & L-STEP & L-STEP & M-STEP \\
MED & L-STEP & L-STEP & L-STEP \\
FAR & & & \\
\hline \hline TABLE II: RULE BASE FOR CHANGE IN RIGHT SERVO POSITION \\
\hline \hline L-SENSOR/ & NEAR & MED & FAR \\
R-SENSOR & S-STEP & L-STEP & L-STEP \\
NEAR & S-STEP & M-STEP & L-STEP \\
MED & S-STEP & M-STEP & L-STEP \\
\hline \hline
\end{tabular}

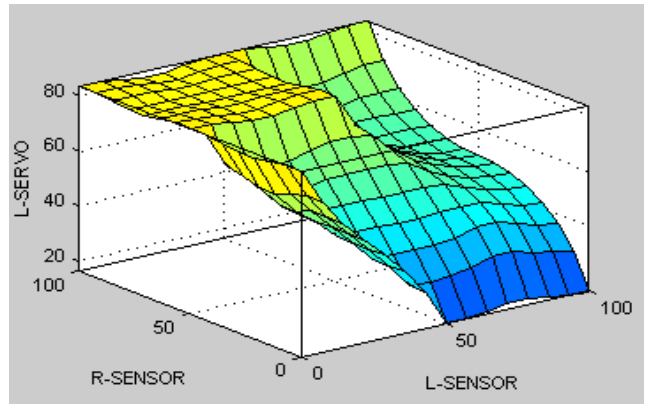

Fig. 6. Left Servo Control Surface.

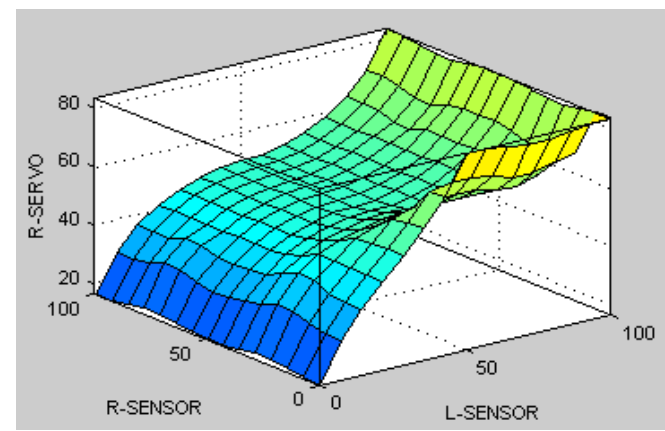

Fig. 7. Right Servo Control Surface.

\section{Fuzzy Implication}

Fuzzy implication helps to evaluate the consequent part of each rule. Among the various implication methods available in literature, Mamdani implication method is selected. After the inputs have been fuzzified and FLC know the degree to which each part of the antecedent of a rule has been satisfied, degree of fulfillment of (DOF) of each rule is calculated using AND operator. The output membership function is then truncated at DOF level. All the rules are evaluated in this 
manner and final output membership functions are aggregated in a cumulative manner using OR operator to yield the final fuzzy output. An example of the implication process is shown in Fig. 8 where L-SENSOR measures FAR and R-SENSOR measures NEAR, so hexapod will start walking towards left to avoid the obstacle in front of R-SENSOR.

\section{Defuzzification}

The result of the implication and aggregation step is the fuzzy output, which is the union of all the individual rules that are validated or fired. Conversion of this fuzzy output to crisp output is defined as defuzzification.

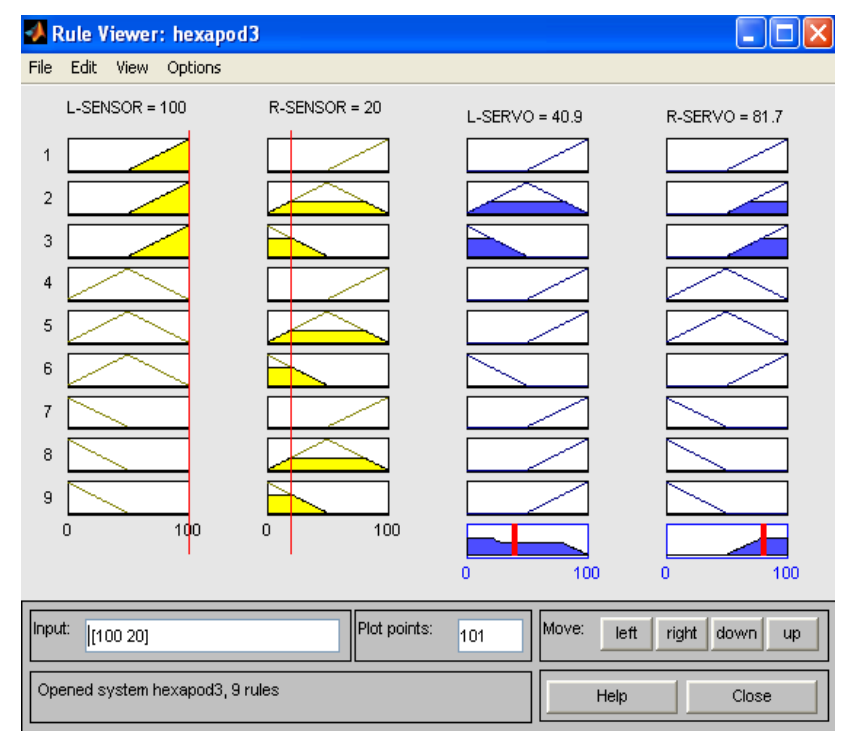

Fig. 8. Implication and Defuzzification Process

Commonly used defuzzification methods are maximum defuzzification and centroid defuzzification. COA method has been used for this purpose and is described as:

$$
Z_{o}=\frac{\sum_{i=1}^{n} Z_{i} \mu_{\text {out }}\left(Z_{i}\right)}{\sum_{i=1}^{n} \mu_{\text {out }}\left(Z_{i}\right)}
$$

where $\mu_{\text {out }}\left(Z_{i}\right)$ are the $i=1,2, \ldots, n$ sampled values of the aggregated output membership function and $Z_{o}$ is the crisp value which describes the duty cycle variation of PWM signal for controlling the position of servo motors.

\section{CONTROLLER IMPLEMENTATION AND RESULTS}

The FLC is implemented using a single chip AT89C52 microcontroller. After the distances to the hurdles are acquired using ultrasonic sensors, fuzzification is performed to transform these crisp values into degree of belongingness. The fuzzification step is a set of three routines including 'mnear(unsigned char val)', 'mmed(unsigned char val)' and 'mfar(unsigned char val)' defined for three intervals which results in mapping the two sensor crisp values in six degree of memberships namely 'lsnear', 'lsmed', 'lsfar', 'rsnear', 'rsmed' and 'rsfar'. After the fuzzification stage, rules are evaluated in sequence. For the execution of each rule, the degree of freedom of that rule is first determined using the 'min(lsmem,rsmem)' routine where the arguments are the degree of memberships of the sensor values to particular fuzzy sets defined for that rule. The output fuzzy sets in that rule are then truncated at the degree of freedom level. The result of this step is saved in an array of 50 characters for each of left and right motor namely 'lmarr' and 'rmarr' using the routines 'slow(unsigned char val, unsigned char arr[])', 'med(unsigned char val, unsigned char arr[])', 'fast(unsigned char val, unsigned char arr[])' where the arguments denote the $\alpha$-cut on the output fuzzy sets and array name respectively. After all the rules have been executed, the arrays contain the final fuzzy sets. The defuzzification is performed on the array contents to yield the final crsip outputs fro the servo motors. The entire process is completed in less than 500ms time frame which is sufficient for real time navigation of hexapod amongst obstacles. The execution time can be further reduced if separate microcontroller is used for each servo. A walking motion of hexapod in an environment containing obstacles is shown in Fig. 9 which validates the proposed controller for obstacle avoidance task.

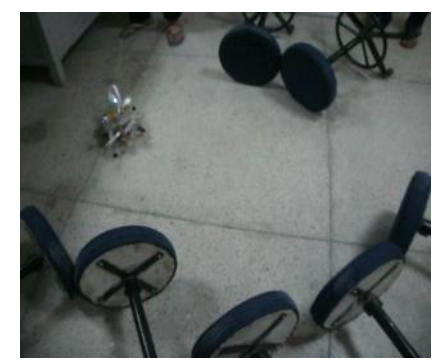

(a)

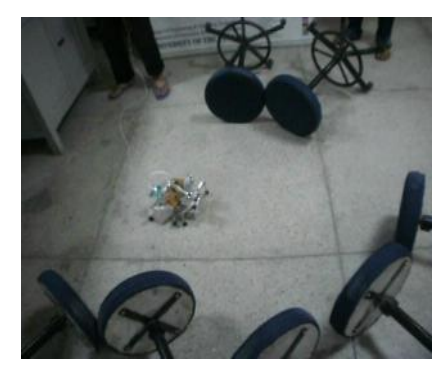

(b)

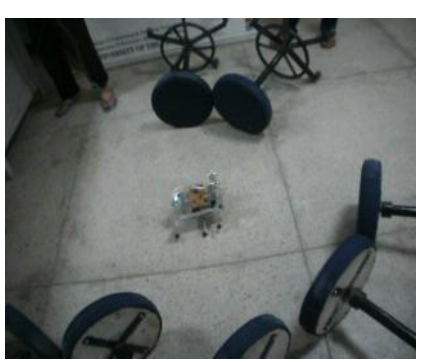

(c)

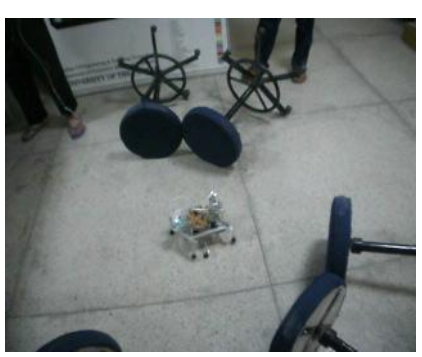

(d) 


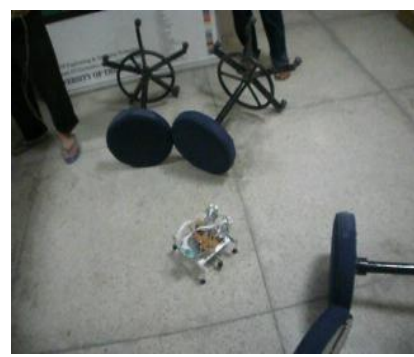

(e)

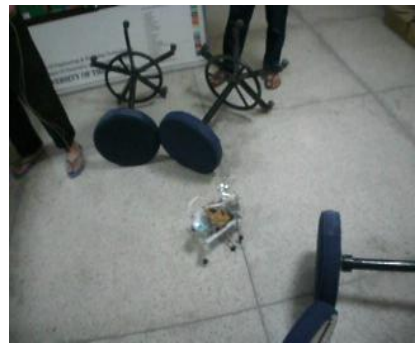

(f)

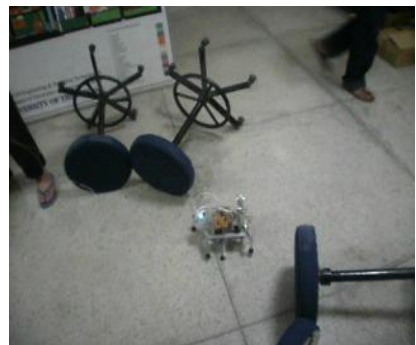

(g)

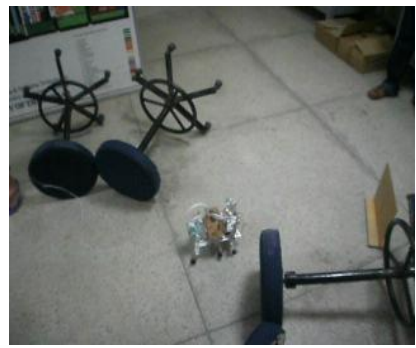

(h)

Fig. 9 (a)-(h) Hexapod walking motion amongst obstacles

\section{CONCLUSIONS}

In this paper, design of a fuzzy logic based obstacle avoidance controller for hexapod walking robot is presented. The designed controller is a two input, two output system and is implemented with cheap AT89C52 microcontroller. The controller shows satisfactory result in presence of obstacles and hexapod can navigate through the obstacles without hitting them. The design is modular i.e., more modules can be easily added without changing the system much.

\section{REFERENCES}

[1] T. Bräunl, Embedded Robotics, Mobile Robot Design and Applications with Embedded Systems, $2^{\text {nd }}$ ed., Springer-Verlag Berlin Heidelberg 2003, 2006.

[2] M. Raibert, "Legged robots," Communications of the ACM 29, vol. 29, issue 6, pp. 499-514, 1986.

[3] F. Delcomyn and M. E. Nelson, "Architectures for a biomimetic hexapod robot," Robotics and Autonomous Systems Journal, vol. 30 , issue 1-2, pp. 5-15, 2000.
[4] D. K. Pratihar, K. Deb, and A. Ghosh, "Optimal path and gait generations simultaneously of a six-legged robot using a GA-fuzzy approach," Robotics and Autonomous Systems Journal, vol. 41, issue 1, pp. 1-20, 2002.

[5] G. McComb, The Robot Builder's Bonanza, $2^{\text {nd }}$ ed., McGraw Hill, 2001.

[6] Jahanzeb, "Design and implementation of an intelligent controller for a walking hexapod robot,” M. S. Thesis, UET Lahore, 2007.

[7] K. Williams, Insectronics: Build Your Own Walking Robot, McGraw-Hill, 2003.

[8] J. S. Roger Jang and N. Gulley, MATLAB Fuzzy Logic Toolbox: A User's Guide, Mathworks Inc., 1997.

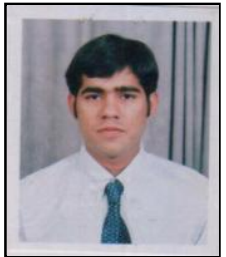

Umar Farooq did his B.Sc. and M.Sc. both in Electrical Engineering from University of Engineering \& Technology Lahore in 2004 and 2010 respectively. $\mathrm{He}$ is currently with the Department of Electrical Engineering, University of The Punjab Lahore. His research interests include the application of intelligent techniques to problems in control engineering, robotics and power electronics.

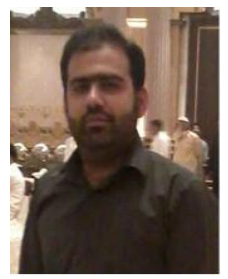

Muhammad Usman Asad did his B.Sc. in Electrical Engineering from University of The Punjab Lahore in 2010. During his stay at Electrical Engineering Department University of The Punjab Lahore, he served as President of Society of Engineering Excellence (2009) and contributed in the research activities of the society. He is the recipient of Gold Medal award for his paper on Ball Scoring Robot in $24^{\text {th }}$ IEEEP International Multi-topic Symposium, 2009 and Silver Medal award for his paper on Neural Controller for Robot Navigation in $26^{\text {th }}$ IEEEP International Multi-topic Symposium, 2011. He is currently working towards M.Sc. degree in Electrical Engineering from G.C. University Lahore. He is with Department of Electrical Engineering, The University of Lahore where he is a Lecturer. His research interests include intelligent control of Robotics and Power systems.

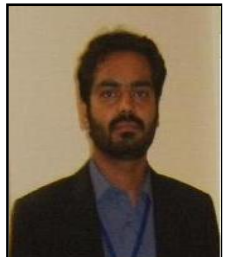

Muhammad Amar did his B.Sc. in Electrical Engineering from University of The Punjab Lahore in 2010 and M.Sc. in Electrical Engineering from University of Engineering \& Technology Lahore in 2012. He is currently working towards Ph.D. degree in Electrical Engineering from Monash University, Australia. His research interests include the application of intelligent techniques to problems in control engineering, robotics and machine vision.

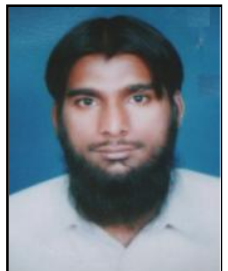

Athar Hanif holds B.Sc. and M.Sc. degrees in Electrical Engineering from University of Engineering $\&$ Technology Taxila and University of Engineering \& Technology Lahore respectively. $\mathrm{He}$ is currently working towards the Ph.D. degree in Control Engineering from Muhammad Ali Jinnah University Islamabad. $\mathrm{He}$ is with Department of Electrical Engineering, The University of Lahore where he is working as Assistant Professor. His research interests include the robust nonlinear control of hybrid vehicles and power converters.

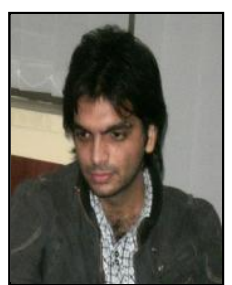

Syed Omar Saleh holds B.Sc. degree in Electrica Engineering from University of The Punjab Lahore. During his stay at Electrical Engineering Department University of The Punjab Lahore, he served as President of Society of Engineering Excellence (2011) and contributed in research activities of the society. $\mathrm{He}$ won the best research paper award twice in IET All Pakistan Electrical Engineering Conferences in 2010 and 2011 held at Ghulam Ishaq Khan Institute of Engineering Sciences for his papers on Fuzzy Logic and Neural Control of Robots and silver medal in $26^{\text {th }}$ IEEEP International Multi-topic Symposium, 2011. His research interests include the intelligent control of Mechatronic and Power systems. 